\title{
Stereotactic body radiotherapy of lymph node metastases under MR-guidance: First clinical results and patient-reported outcomes
}

\author{
Fabian Weykamp ${ }^{1,2,3} \cdot$ Charlotte Herder-Wagner ${ }^{1}$ Sebastian Regnery',2,3 Philipp Hoegen 1,2,3,5

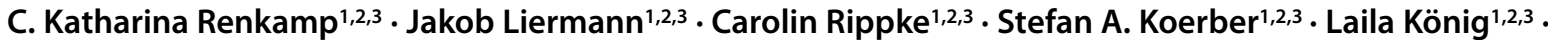 \\ Carolin Buchele $^{1,2,3} \cdot$ Sebastian Klüter ${ }^{1,2,3} \cdot$ Jürgen Debus $\mathbf{S}^{1,2,3,4,5,6}$. Juliane Hörner-Rieber ${ }^{1,2,3,5}$ (D)
}

Received: 12 February 2021 / Accepted: 1 August 2021 / Published online: 1 September 2021

(c) The Author(s) 2021

\begin{abstract}
Objective Stereotactic body radiotherapy (SBRT) is a noninvasive treatment option for lymph node metastases (LNM). Magnetic resonance (MR)-guidance offers superior tissue contrast and enables treatment of targets in close vicinity to radiosensitive organs at risk (OAR). However, literature on MR-guided SBRT of LNM is scarce with no report on outcome parameters.

Materials and methods We report a subgroup analysis of a prospective observational study comprising patients with LNM. Patients received MR-guided SBRT at our MRIdian Linac (ViewRay Inc., Mountain View, CA, USA) between January 2019 and February 2020. Local control (LC), progression-free survival (PFS) and overall survival (OS) analysis were performed using the Kaplan-Meier method with log rank test to test for significance $(p<0.05)$. Our patient-reported outcome questionnaire was utilized to evaluate patients' perspective. The CTCAE (Common Terminology Criteria for Adverse Events) v. 5.0 was used to describe toxicity.

Results Twenty-nine patients (72.4\% with prostate cancer; $51.7 \%$ with no distant metastases) received MR-guided SBRT for in total $39 \mathrm{LNM}$. Median dose was $27 \mathrm{~Gy}$ in three fractions, prescribed to the $80 \%$ isodose. At 1-year, estimated LC, PFS and OS were 92.6, 67.4 and $100.0 \%$. Compared to baseline, six patients $(20.7 \%)$ developed new grade I toxicities (mainly fatigue). One grade II toxicity occurred (fatigue), with no adverse event grade $\geq$ III. Overall treatment experience was rated particularly positive, while the technically required low room temperature still represents the greatest obstacle in the pursuit of the ideal patient acceptance.

Conclusion MR-guided SBRT of LNM was demonstrated to be a well-accepted treatment modality with excellent preliminary results. Future studies should evaluate the clinical superiority to conventional SBRT.
\end{abstract}

Keywords Pelvis - Patient acceptance $\cdot$ Abdomen $\cdot$ Organs at risk · Visability

\section{Background and purpose}

Stereotactic body radiotherapy (SBRT) enables the application of high tumoricidal irradiation doses while simultaneously sparing organs at risk (OAR). Through local-

Data Availability The datasets generated for this study are available on request to the corresponding author.

PD Dr. med. Juliane Hörner-Rieber

juliane.hoerner-rieber@med.uni-heidelberg.de

1 Department of Radiation Oncology, Heidelberg University Hospital, Heidelberg, Germany

2 Heidelberg Institute of Radiation Oncology (HIRO), Heidelberg University Hospital, Heidelberg, Germany ized radiotherapy, systemic therapy might be postponed to improve quality of life [1]. Nonetheless, especially when treating lymph node metastases (LNM) in the abdomen or pelvis, surrounding intestines are put in jeopardy [2]. Especially lymph node metastases of prostate cancer are lo-
3 National Center for Tumor diseases (NCT), Heidelberg University Hospital, Heidelberg, Germany

4 Heidelberg Ion-Beam Therapy Center (HIT), Departmen of Radiation Oncology, Heidelberg University Hospital, Heidelberg, Germany

5 Clinical Cooperation Unit Radiation Oncology, German Cancer Research Center (DKFZ), Heidelberg, Germany

6 core center Heidelberg, German Cancer Consortium (DKTK), Heidelberg, Germany 
calized more frequently around or abut intestinal structures [3].

During the last few years, there has been a growing interest in SBRT of LNM, as high local control rates and favorable toxicity profiles have been reported [4]. However, standard image-guided SBRT via cone beam CT scan (CBCT) only offers limited soft tissue contrast [5]. Furthermore, counterintuitively, for SBRT of LNM, respiratory motion also has to be accounted for in some cases [6-9]. This can be conventionally achieved by using an internal target volume (ITV) concept, which nonetheless results in a larger target volume leading to a higher dose load within OAR [10]. Online MR-guided radiotherapy is a relatively new treatment solution and offers superior soft tissue contrast, with some systems being also able to provide gated dose delivery [11]. Thus, the target volume and OAR can be monitored live during beam-on time. Due to the relatively long treatment sessions with MR-guided radiotherapy, one should also be aware of patient acceptance [12].

Available data on MR-guided SBRT of LNM are scarce. Moreover, to the best of our knowledge, no clinical results are available on MR-guided radiotherapy of these patients, except for a single case report [13]. The purpose of our study was to provide first outcome data and report on patient acceptance of this novel treatment modality.

\section{Methods}

The study presented here is a subgroup analysis of a prospective observational trial and comprises cancer patients with lymph node metastases treated with MRguided SBRT using the MRIdian Linac (ViewRay Inc., Mountain View, CA, USA) between January 2019 and February 2020. SBRT was defined as a single fraction dose $\geq 4$ Gy and a number of fractions $\leq 12$, in accordance with the current guideline of the working group "Stereotactic Radiotherapy" of the German Society of Radiation Oncology [14].

\section{Treatment characteristics}

A thorough description of our treatment simulation and treatment planning has been published previously [12]. Five patients in our presented study were also part of the respective publication. In short, treatment simulation at the MR-Linac started with the acquisition of three-dimensional (3D) MR images in either inspiration breath-hold or free breathing using the true fast imaging with steady-state precession sequence (TrueFISP), followed by planar cine-MRI in a sagittal plane to evaluate motion of the target structure. The acquired MR image data were used as the primary image set for treatment planning. The acquisition of image data at the MR-Linac also functioned as a first check for patients' compliance.

Afterwards, a planning CT scan with contrast-enhanced and noncontrast-enhanced sequences was acquired in the planned treatment position. The gross tumor volume (GTV) was defined as the macroscopic tumor volume in the MRI scan and the coregistered CT scan. As proposed by the current German guidelines for patients with recurrent prostate cancer, all prostate cancer patients had undergone a prostate-specific membrane antigen positron emission tomography CT scan (PSMA-PET-CT) before treatment simulation using the MR-Linac [15]. Thus, GTV of all prostate cancer patients was additionally defined on the PSMA-PET-CT. Clinical target volume (CTV) equaled the GTV with additional $2 \mathrm{~mm}$ adapted to natural organ boundaries. Typical planning target volume (PTV) margins for CT-based SBRT of LNM are 5-7 mm [16, 17]. We chose a PTV margin of $3 \mathrm{~mm}$, which has been established in this setting for MR-Linac treatment $[18,19]$.

Every day, image guidance was carried out via the onboard 3D MRI with identical settings (field of view, duration, pulse sequence, breathing instructions) as during the original MR simulation. Soft-tissue-based registration with the reference MR scan was applied and always registered directly on the GTV. For MR-gating in real time, the TrueFISP sequence was applied using one sagittal slice (four frames per second). If the lymph node metastasis was detectable on the TrueFISP sequence, the lesion was used directly as the gating structure (the region of interest [ROI]). In any other case, an anatomical surrogate structure surrounding the target lesion was defined as the gating structure. Then, a gating boundary was created by applying a ROI expansion of $3 \mathrm{~mm}$. The irradiation process was interrupted if the target structure left the tolerance field around the gating boundary (set to $3 \%$, if necessary up to $5 \%$ in exceptional cases). Patients were offered additional visual control during the gating process through an in-room monitor showing the live sagittal cine-MRI and could thus modulate their breathing [12]. For demonstration purposes, a video of this process can be found in the supplementary material section. In our described patient collective, no online treatment adaptation was performed, as it had not yet been implemented.

Prescribed irradiation doses were chosen depending on the size of the target volume as well as the proximity to organs at risk. If possible, three fractions of $9 \mathrm{~Gy}$ were used, prescribed to the conformally enclosing $80 \%$ isodose, covering at least $95 \%$ of the PTV. Target volumes in close proximity to radiosensitive structures (e.g. the small bowel) were irradiated with six fractions of $5 \mathrm{~Gy}$ prescribed to the conformally enclosing $80 \%$ isodose. One larger para-aortic metastasis was treated with five fractions of $10 \mathrm{~Gy}$ prescribed to the conformally enclosing $80 \%$ isodose, since 
there was no dose-limiting OAR in the proximity. Target coverage was reduced in case OAR dose constraints could not be met. Dose constraints were the following (for three fractions) and adopted from Hanna et al. [20]:

- Esophagus: $0.5 \mathrm{cc}<25.2 \mathrm{~Gy}$

- Stomach/Duodenum: $0.5 \mathrm{cc}<22.2 \mathrm{~Gy}$

- Small bowel: $0.5 \mathrm{cc}<25.2 \mathrm{~Gy}$

- Sigma/Rectum: $0.5 \mathrm{cc}<28.2 \mathrm{~Gy}$

- Kidney: Mean dose $<8.5 \mathrm{~Gy}$

- Spinal cord: $0.1 \mathrm{cc}<21.6 \mathrm{~Gy}$

- Cauda equina: $0.1 \mathrm{cc}<24 \mathrm{~Gy}$

- Central airways: $0.5 \mathrm{cc}<32 \mathrm{~Gy}$

- Heart: $0.5 \mathrm{cc}<26 \mathrm{~Gy}$

- Ureter: $0.5 \mathrm{cc}<40 \mathrm{~Gy}$

- Bladder: $0.5 \mathrm{cc}<28.2 \mathrm{~Gy}$

- Femoral heads: $10 \mathrm{cc}<21.9 \mathrm{~Gy}$
A self-developed patient-reported outcome questionnaire (PRO-Q) was used to evaluate patients' experience with the MR-Linac treatment (graded from 1-5, where 1 represents a completely positive and 5 a completely negative experience) [12]. In addition, our MR-Linac staff was surveyed about their judgement on each patient's treatment performance (graded from 1-10, where 1 represents a facile and 10 a nearly inacceptable expenditure).

\section{Endpoints and statistical methods}

Local control (LC), progression-free survival (PFS) and overall survival (OS) were calculated starting from the first day of the SBRT. LC was evaluated per lesion, whereas PFS and OS were calculated per patient. Toxicity was described using the Common Terminology Criteria for Adverse Events (CTCAE v. 5.0).

Table 1 Patient characteristics $(n=29)$

\begin{tabular}{|c|c|c|}
\hline Median age & 70 years & Range $37-80$ years \\
\hline Median Karnofsky score & $90 \%$ & Range $80-100 \%$ \\
\hline Median body mass index & $27.1 \mathrm{~kg} / \mathrm{m}^{2}$ & Range $21.2-35.2 \mathrm{~kg} / \mathrm{m}^{2}$ \\
\hline Female/male & $2 / 27$ & $6.9 \% / 93.1 \%$ \\
\hline Prostate cancer & 21 & $72.4 \%$ \\
\hline Other & 8 & $27.6 \%$ \\
\hline \multicolumn{3}{|c|}{$\begin{array}{l}n=2 \text { gastric cancer, } n=2 \text { colorectal carcinoma, } n=1 \text { adenoid cystic carcinoma, } n=1 \text { urinary bladder carcinoma, } n=1 \text { renal carcinoma, } n=1 \text { tub } \\
\text { cancer }\end{array}$} \\
\hline No distant metastases present & 15 & $51.7 \%$ \\
\hline Oligometastatic disease ( $n \leq 3$ distant metastases) & 10 & $34.5 \%$ \\
\hline Oligoprogressive disease ( $n>3$ distant metastases) & 4 & $13.8 \%$ \\
\hline Extranodal disease progression within 4 weeks before irradiation & 3 & $10.3 \%$ \\
\hline \multicolumn{3}{|l|}{$n=2$ prostate cancer, $n=1$ tubal cancer } \\
\hline Systemic therapy within 4 weeks before irradiation & 9 & $31.0 \%$ \\
\hline \multicolumn{3}{|l|}{$n=6$ hormonal therapy, $n=3$ chemotherapy } \\
\hline Systemic therapy within 4 weeks after irradiation & 7 & $24.1 \%$ \\
\hline \multicolumn{3}{|l|}{$n=6$ hormonal therapy, $n=1$ chemotherapy } \\
\hline \multicolumn{3}{|l|}{ Adverse events before radiotherapy } \\
\hline $\begin{array}{l}\text { Grade I } \\
\text { (Including: fatigue, pain, constipation, flatulence, nycturia, diarrhea, } \mathrm{n}\end{array}$ & 12 & $41.4 \%$ \\
\hline \multicolumn{2}{|l|}{ (Proctitis) } & $3.4 \%$ \\
\hline Grade $\geq I I I$ & 0 & 0 \\
\hline \multicolumn{3}{|l|}{ Adverse events at last treatment day } \\
\hline \multicolumn{3}{|c|}{ (Including: fatigue, pain, constipation, flatulence, nycturia, diarrhea, nausea, cough) } \\
\hline \multicolumn{3}{|l|}{ (Fatigue) } \\
\hline Grade $\geq I I I$ & 0 & 0 \\
\hline \multicolumn{3}{|l|}{ Adverse events at first follow-up } \\
\hline \multicolumn{2}{|l|}{ (Including: fatigue, flatulence, dyspnea) } & $13.8 \%$ \\
\hline Grade II & 0 & 0 \\
\hline Grade $\geq I I I$ & 0 & 0 \\
\hline
\end{tabular}


Table 2 Irradiation treatment characteristics

\begin{tabular}{|c|c|c|}
\hline \multicolumn{3}{|c|}{$\begin{array}{l}\text { Total number of irradiated lymphatic nodes per patient }(n=39 \text { le- } \\
\text { sions) }\end{array}$} \\
\hline$n=1$ & 20 & $69.0 \%$ \\
\hline$n=2$ & 8 & $27.6 \%$ \\
\hline$n=3$ & 1 & $3.4 \%$ \\
\hline $\begin{array}{l}\text { Localization of irradiated } \\
\text { lymphatic nodes }\end{array}$ & \multicolumn{2}{|c|}{$\begin{array}{l}\text { Mediastinal }(14.7 \%) \text {, thoracic } \\
\text { aorta }(2.9 \%) \text {, retroperitoneal } \\
(14.7 \%) \text {, pelvis }(67.7 \%)\end{array}$} \\
\hline Target volumes $(n=34)$ & Median & Range \\
\hline GTV & $1.8 \mathrm{~mL}$ & $0.1-70.8 \mathrm{~mL}$ \\
\hline$C T V$ & $3.3 \mathrm{~mL}$ & $0.4-81.7 \mathrm{~mL}$ \\
\hline$P T V$ & $10.0 \mathrm{~mL}$ & $2.6-110.3 \mathrm{~mL}$ \\
\hline Prescribed total dose & $27 \mathrm{~Gy}$ & 24-50Gy \\
\hline Fractions & 3 & $3-6$ \\
\hline GTV D50 & $33.0 \mathrm{~Gy}$ & $23.6-51.6 \mathrm{~Gy}$ \\
\hline \multicolumn{3}{|c|}{ EQD2 and BED for prostate histology $(n=26)$} \\
\hline$E Q D 2(\alpha / \beta=3)$ & $64.8 \mathrm{~Gy}$ & 48.0-64.8 Gy \\
\hline$B E D(\alpha / \beta=3)$ & $108.0 \mathrm{~Gy}$ & 80.0-108.0 Gy \\
\hline \multicolumn{3}{|c|}{ EQD2 and BED for other histology $(n=8)$} \\
\hline$E Q D 2(\alpha / \beta=10)$ & $37.5 \mathrm{~Gy}$ & $37.5-130.0 \mathrm{~Gy}$ \\
\hline$B E D(\alpha / \beta=10)$ & $45.0 \mathrm{~Gy}$ & $45.0-216.7 \mathrm{~Gy}$ \\
\hline Treatment time ("on table") & $43.0 \mathrm{~min}$ & $26.0-93.0 \mathrm{~min}$ \\
\hline Radiation time & $11.0 \mathrm{~min}$ & $7.0-26.0 \mathrm{~min}$ \\
\hline Beam on time per fraction & $3.5 \mathrm{~min}$ & $1.7-5.0 \mathrm{~min}$ \\
\hline
\end{tabular}

$B E D$ biologically effective dose, $C T V$ clinical target volume, $E Q D 2$ equivalent dose at $2 \mathrm{~Gy}, G T V$ gross tumor volume, $P T V$ planning target volume

Following the study protocol, each patient was specifically assessed for presence of fatigue, nausea, vomiting, diarrhea, constipation, dyspnea, cough, skin disorder and pain. This evaluation took place before irradiation, at the last treatment day and at first follow-up. Patients with prostate cancer received a prostate-specific antigen (PSA) measurement 6-8 weeks after SBRT and then every 3 months. In case of a rising PSA level in two consecutive measures, patients were evaluated for receiving a new PSMA-PET scan as offered by current German guidelines to identify the exact location of the recurrence [15]. The other patients received a follow-up with a contrast enhanced MRI or CT scan, performed 6-8 weeks after the SBRT as well as a clinical examination. Further clinical and imaging follow-up was performed every 3 months at the discretion of the responsible oncologist and was not part of the prospective study.

LC, PFS and OS were estimated with the Kaplan-Meier method. Univariate analysis was performed with the logrank method to test for significance. Median follow-up time was assessed using the reverse Kaplan-Meier method. The biologically effective dose (BED) was calculated applying the linear-quadratic model [21]. An $\alpha / \beta$ ratio of 3 was assumed for LNM of prostate origin, an $\alpha / \beta$ ratio of 10 was as- sumed for all other origins [3]. Statistical analysis was performed with SPSS software (Version 24.0, IBM, Armonk, NY, USA). A $p$-value of $<0.05$ was defined as significant. The MR-Linac observational study was approved by the ethics committee of the University Hospital (S-543/2018).

\section{Results}

Median patient age was 70 years. Most patients treated had a very good Karnofsky performance status. The most common primary tumor was prostate cancer $(72.4 \%)$, of which $66.7 \%$ had no further metastases and $33.3 \%$ had 1 to 3 further metastases, reflecting the lower metastastic burden in this patient group. Systemic therapy was present in $31.0 \%$ of the patients before and in $24.1 \%$ of the patients after SBRT. In prostate cancer patients, $28.6 \%$ had antihormonal therapy before and $28.6 \%$ after SBRT. Further patient characteristics are displayed in Table 1.

Treatment characteristics are described in Table 2. Most patients received SBRT of a single lymphatic metastasis (69.0\%), which was mainly located in the pelvis $(67.7 \%)$. Median GTV and CTV size were $1.8 \mathrm{~mL}(0.1-70.8 \mathrm{~mL})$ and $3.3 \mathrm{~mL}(0.4-81.7 \mathrm{~mL})$ with a median prescription dose of $27 \mathrm{~Gy}$ (24-50 Gy). Fig. 1 shows a typical treatment plan of the pelvis. Moreover, it demonstrates the high soft-tissue contrast, enabling to distinct the treatment volume from the neobladder (yellow line) in the sagittal plane.

\section{Outcome}

Median follow-up was 13.0 months. Estimated LC was 92.6\% at 12 months (Fig. 2a). Three patients suffered from local recurrence ( $n=1$ prostate cancer, $n=1$ colon carcinoma, $n=1$ urinary bladder cancer). In these three cases, the irradiated LNM itself had recurred. One additional patient had nodal recurrence (prostate cancer), at distance from the irradiated LNM. PFS at 12 months was $67.4 \%$ (Fig. 2b) and was higher in prostate cancer patients than in nonprostate cancer patients $(83.3 \%$ vs. $14.6 \%, p<0.01$; Fig. 2 c). One patient died during follow-up. Estimated OS at 12 months was $100.0 \%$ (Fig. 2d).

\section{Toxicity}

For detailed toxicity data, please see Table 1 . Nearly half of the patients already had mild complaints before starting SBRT (43.4\%; CTC grade I-II). Compared to baseline, 6 patients $(20.7 \%)$ developed new grade I toxicities on the last day of radiotherapy (fatigue, pain, constipation, flatulence, nycturia, diarrhea, nausea and/or coughing). One grade II toxicity occurred (fatigue). No adverse event grade III or higher was reported at any time. 
Fig. 1 MR-Linac treatment plan (3 fractions of $9 \mathrm{~Gy}$ prescribed to the conformally enclosing $80 \%$ isodose) from different perspectives ( $I$ inferior, $A$ anterior, $R$ right) with and without isodose lines
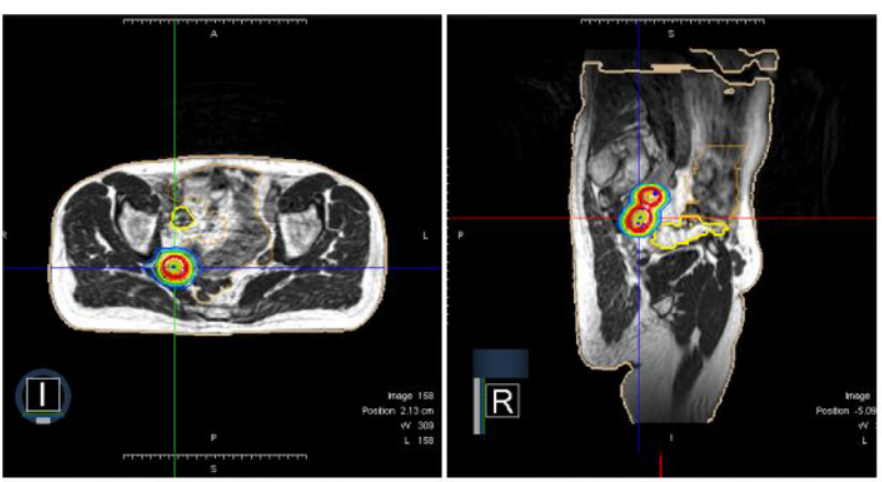

Isodose Lines
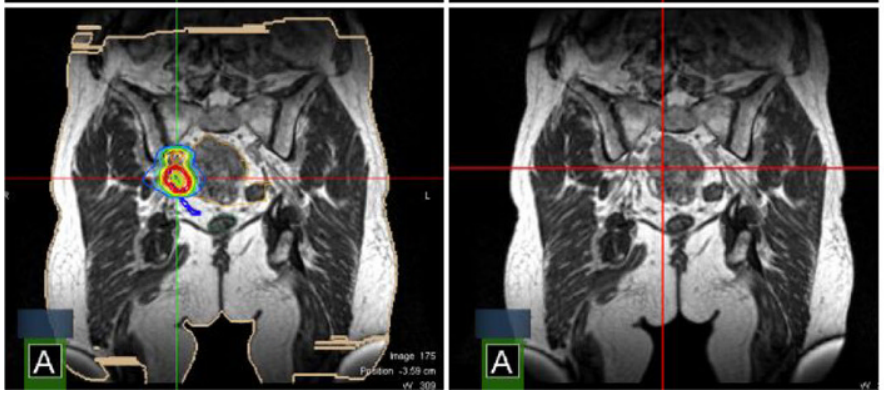

Rx Dose = 27.00 Gy
\begin{tabular}{|c|c|}
\hline Dose (Gy) & Rx (\%) \\
\hline 28.89 & 107.0 \\
\hline 25.65 & 95.0 \\
\hline 24.30 & 90.0 \\
\hline 21.60 & 80.0 \\
\hline 18.90 & 70.0 \\
\hline 16.20 & 60.0 \\
\hline 13.50 & 50.0 \\
\hline
\end{tabular}

a

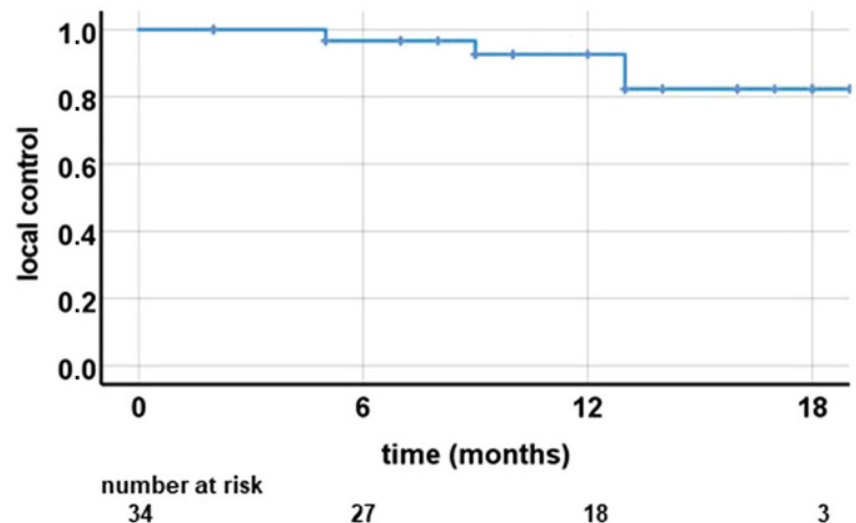

c

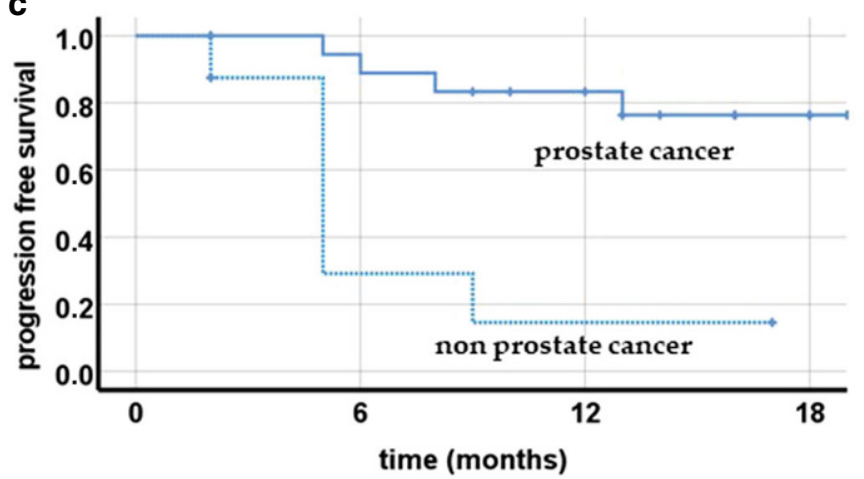

number at risk

29

$2 / 17$ b

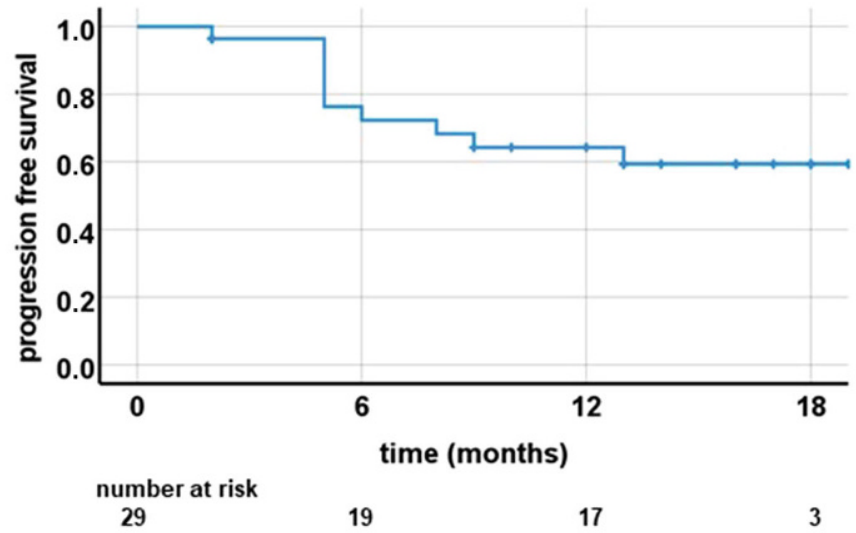

d

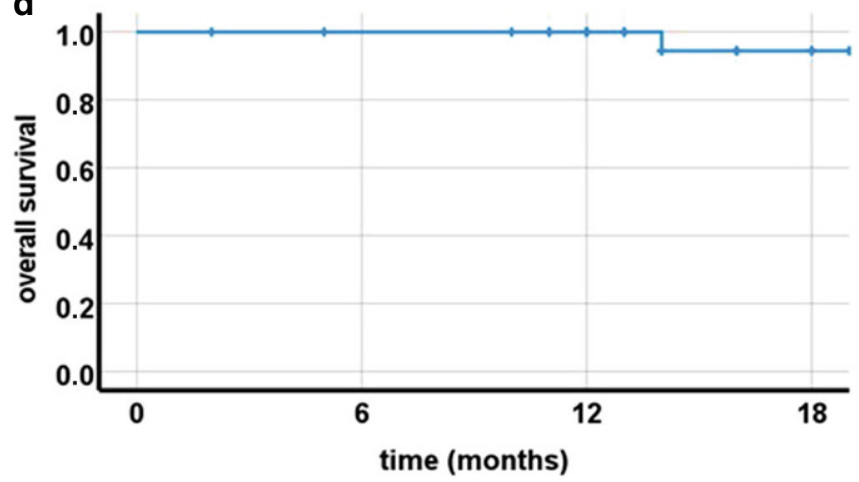

number at risk

29

24

12

Fig. 2 Local control (a), progression-free survival (b) divided by prostate cancer histology (c) and overall survival (d) following magnetic resonance (MR)-guided stereotactic body radiotherapy (SBRT) of lymph node metastases 
Table 3 Patient- (items 1-18) and staff (item 19)-reported outcome (available for $n=23$ patients)

\begin{tabular}{lcl}
\hline & Median & Range \\
\hline $\begin{array}{l}\text { Categorical point scale form 1-5, where 1 equals very positive and } 5 \\
\text { equals very negative }\end{array}$ & 1 & $1-2$ \\
1. Overall treatment experience & 1 & $1-2$ \\
2. Information provided by the staff & 1 & 1 \\
3. Friendliness of the staff & 2 & $1-4$ \\
4. Duration of the treatment & 2 & $1-4$ \\
5. Size of the MRI bore & 2 & $1-5$ \\
6. Positioning during radiotherapy & 2 & $1-3$ \\
7. Having to lie still & 2 & $1-4$ \\
8. Noise in the MR-Linac & 3 & $1-4$ \\
9. Temperature in the MR-Linac & 3 & $1-4$ \\
$\begin{array}{l}\text { 10. Local temperature of body parts } \\
\text { 11. Tingling sensations in fingers and toes }\end{array}$ & 1 & $1-5$ \\
12. Breathing instructions & 1 & $1-3$ \\
13. Breath holding & 1 & $1-3$ \\
14. Anxiousness during treatment session & 1 & $1-3$ \\
15. Reported time until full recovery after \\
the radiotherapy session
\end{tabular}

\section{Patient- and staff-reported outcome}

Table 3 shows patient- and staff-reported outcome. Treatment at the MR-Linac was accepted particularly well, especially overall treatment experience and items regarding the staff were rated very positively (each median 1 point, no rating $>2$ ). The low room temperature was not well tolerated (median 3 points). The median time to physical and mental recovery after the first treatment session were 0 min (range 0-300 min). The MR-Linac staff reported rather low treatment expenditure (median 3 points).

\section{Discussion}

The presented subgroup analysis of a prospective observational study comprises 29 patients with a total of 39 lymph node metastases treated with ablative MR-guided SBRT from January 2019 to February 2020. Most patients were diagnosed with prostate cancer and showed a very good Karnofsky performance status, with a median age of 70 years.
Irradiating lymph node metastases via online MR-guidance has been proven to be feasible [22]. However, data are only available from one other study group evaluating MR-guided SBRT using the 1.5T Elekta MR-Linac: Winkel et al. retrospectively compared high field $(1.5 \mathrm{~T})$ MR-guided irradiation of lymph node metastases with conventional CBCT-Linac treatment in 20 patients and demonstrated fewer unplanned violations of OAR constraints [19, 22]. The same study group also successfully evaluated the utilization of a vacuum cushion for MR-guided pelvic/paraaortic lymph node SBRT to reduce intrafractional motion [23]. However, to the best of our knowledge, there has not yet been any study about clinical results and patient-reported outcome following MR-guided SBRT of lymph node metastases.

By contrast, conventional SBRT of lymph node metastases has been shown to be effective and safe in prostate cancer, with a LC rate at 24 months of $84 \%$ and no grade III toxicity or higher [24]. Another study including patients with prostate cancer LNM described $98 \%$ LC during a median follow-up of 30 months, with only one case of grade III toxicity [25]. CBCT-SBRT of LNM was also proven to be effective for various primary tumors, with LC rates after 1 year of up to $98 \%$ and a comparably favorable side effect profile $[16,17]$. Table 4 summarizes the larger studies on radiotherapy of lymphatic node metastases with CBCTor MR-Linac-guided SBRT. With a median of $27 \mathrm{~Gy}$ in three fractions (median BED 51.3 Gy), our dose prescription appears to be rather lower. However, we performed a prescription to the conformally enclosing $80 \%$ isodose, covering at least $95 \%$ of the PTV, to allow for a steeper dose gradient. This leads to a higher dose in the GTV and a dose maximum of $125 \%$. Most studies in the field use a prescription to the $98 \%$ or $95 \%$ isodose, which does not include a dose escalation in the GTV and impairs a direct comparison of the simple prescription doses.

Treatment toxicity of our cohort was comparable to CBCT-guided SBRT with only one case of grade II toxicity (fatigue) and no grade III toxicity or higher [16, 17, 24]. Local control of the irradiated lymph nodes was high in our study group with a rate of $92.6 \%$ after 12 months, which lies within the range of the aforementioned studies (87.2-97.9\%). PFS was $64.3 \%$ at 12 months. As previously described, prostate cancer patients had a superior PFS to nonprostate cancer patients $(83.3 \%$ vs. $14.6 \%, p<0.01$; Fig. 2c; $[17,26])$. In a systematic review of 211 prostate cancer patients receiving SBRT for LNM, antihormonal therapy was present in $40.5 \%$ of the patients vs. $28.6 \%$ in the presented study. SBRT might offer the possibility to postpone systemic therapy and hence improve quality of life [1].

Although, clinical results of conventional SBRT for LNM are satisfying, further treatment optimization is war- 


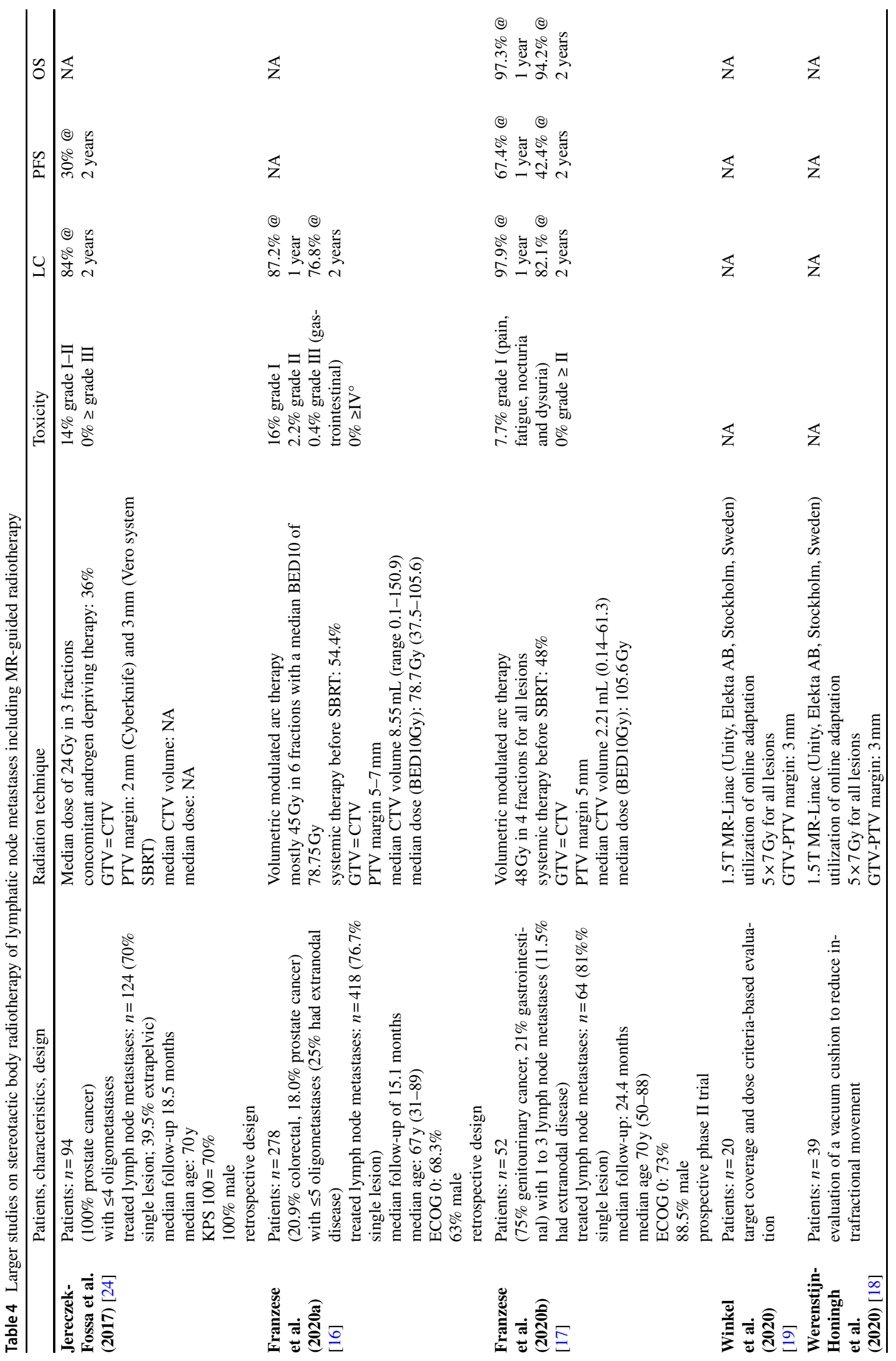




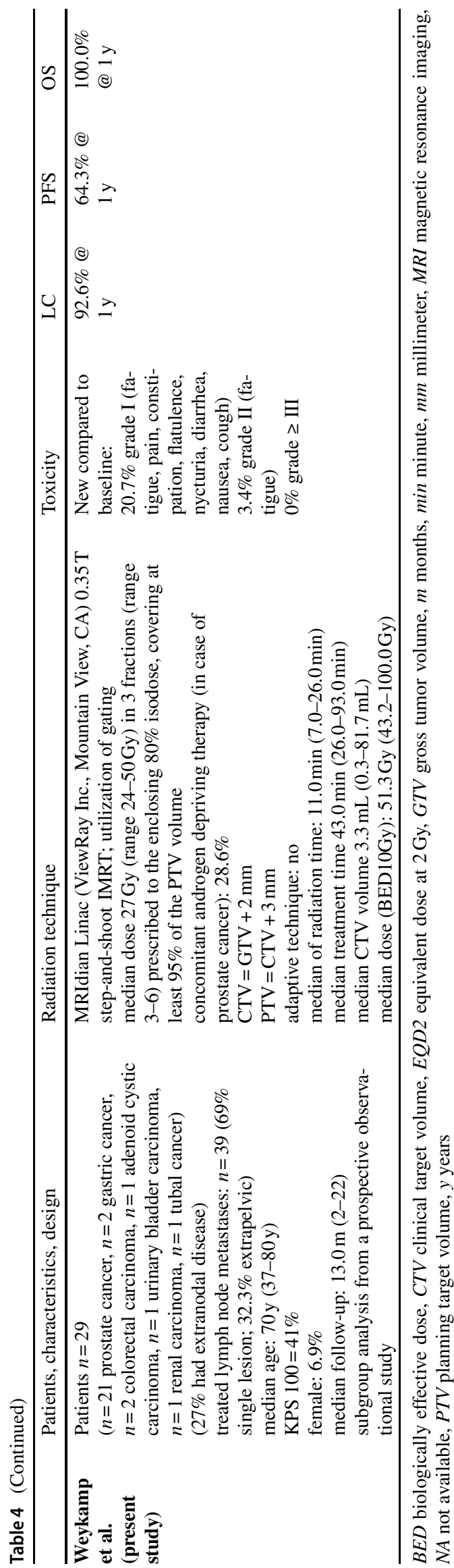

ranted [2]. MR-guided radiotherapy is thought to become a potential practice changing technology in the treatment of various tumor entities, as it offers superior soft tissue contrast for the precise identification of the target volume and detection of inter- and intrafractional changes in adjacent OAR. This new versatile technology thus supports the delivery of high irradiation doses while sparing OAR [27-29]. Gated dose delivery allows for tighter OAR margins and further reduces the proportion of irradiated healthy tissue [10]. About two third (67.7\%) of the irradiated lymph nodes metastases in our study cohort were localized in the pelvis. Nonetheless, we performed gated dose delivery also in these patients to gain insight into breathing motion, which then played a minor role in daily practice.

Despite a rather long treatment time (median $43.0 \mathrm{~min}$ ) lying on a nonpadded treatment couch, patients reported an excellent overall treatment experience, with no rating being below the second-best possible grade (Table 3). MRguided radiotherapy is staff and time intensive [27], while beam on time (median $3.5 \mathrm{~min}$ ) represents only a small proportion of the overall treatment time (median $43.0 \mathrm{~min}$ ). For technical reasons, temperature is cooled down in the treatment room. Although patients who tend to feel cold easily are allowed to wear nonmetal personal clothes under the standardly worn medical scrubs or are provided with an additional blanket, the low temperature is still the greatest obstacle concerning the pursuit of the ideal subjective treatment experience (median grade 3). Given the described hindering circumstances of long treatment duration, small MRI bore and low room temperature, the patient reported experience with the gating process was rated surprisingly positive (median grade 1, range 1-3). Most patients needed no recovery time after the respective daily treatment sessions. Having the tumor displayed in front on oneself as part of the gating process did in general not cause anxiety in the patients, yet even seemed to provide a feeling of power and control. One patient described this setting as highly satisfying and relieving, being able to act directly and actively against the tumor after several passive months of fearing his tumor to recur.

A limitation of the presented study is its rather small sample size and its, at the present time, short follow-up. Nonetheless, our presented patient cohort is the first study in the field, to the best of our knowledge. Moreover, toxicity data were gathered prospectively, while long-term experience is still immature. Another limitation of this study is the utilization of an in-house designed, not externally validated questionnaire. Our questionnaire was specifically designed for our institution. Next to evaluating patients' satisfaction and feasibility of MR-Linac treatment, we used the tool as a quality assessment instrument to measure our staff's performance and opinion. Since there was no control group, patients could have had a high level of satisfaction in the 
first place. Undeniably, there is an underlying selection bias, with patients being excluded from the study due to claustrophobia or pacemaker devices, which might have influence on the patient-reported outcome.

Online adaptive MR-guided SBRT, a procedure where a new treatment plan is created before each irradiation session based on daily anatomy changes, is to date described for liver, adrenal, pancreatic and lung tumors as well as lymph node metastases [18, 30-37]. Online adaption was implemented into our clinical workflow in February 2020 and has since been used daily for every patient, including patients with lymph node metastases. First experience with high field (1.5 T) MR-guided pelvic/para-aortic lymph node SBRT is promising [19]. The PTV coverage was herein shown to be higher with an adaption to the shape of the daily anatomy rather than with an adaption to the mere patients' position [38]. Nonetheless, it needs to be kept in mind that adaption will further prolong the already demanding treatment procedure.

However, also without daily adaption, MR-guided SBRT enables the visualization of the target volume itself as well as the surrounding OAR. Hence, also LNM close to critical OAR can be visually separated through the high soft-tissue contrast of the MRI and treated safely (Fig. 1).

\section{Conclusion}

MR-guided SBRT of lymph node metastases represents a particularly well-accepted treatment modality as measured by our patients' questionnaire. Local control was excellent with only mild toxicity. Our results confirm the need for prospective studies to identify patients, in which OAR would otherwise have hindered SBRT.

Video online The online version of this article contains one video. The article and the video are available online (https://doi.org/10.1007/ s00066-021-01834-w). The video can be found in the article back matter as "Supplementary Information".

Author Contribution FW made the statistical analysis and drafted the manuscript. CHW and FW performed the data collection. SAK, $\mathrm{PH}, \mathrm{LK}, \mathrm{SR}$, JL, SK helped with data collection as well as figure and table preparation. JHR, SK, CKR, CR and CB performed patient treatment. JHR and JD participated in the study design and helped to draft the manuscript. All the authors were responsible for data interpretation, participated in manuscript revisions, and approved the final manuscript.

Funding Open Access funding enabled and organized by Projekt DEAL.

Conflict of interest S.A. Koerber reports grants from Viewray Inc., outside the submitted work. S. Klüter has received personal fees and travel reimbursement from Viewray Inc. J. Debus received grants from CRI-The Clinical Research Institute GmbH, View Ray Inc., Accuray International, Accuray Incorporated, RaySearch Laboratories AB, Vision RT limited, Astellas Pharma GmbH, Merck Serono GmbH, As- tra Zeneca GmbH, Solution Akademie GmbH, Ergomed PLC Surrey Research Park, Siemens Healthcare GmbH, Quintiles GmbH, Pharmaceutical Research Associates GmbH, Boehringer Ingelheim Pharma GmbH Co, PTW-Freiburg Dr. Pychlau GmbH, Nanobiotix A.A. as well as IntraOP Medical outside the submitted work. J. Hörner-Rieber received speaker fees and travel reimbursement from ViewRay Inc., as well as travel reimbursement form IntraOP Medical and Elekta Instrument AB outside the submitted work. F. Weykamp, C. Herder-Wagner, S. Regnery, P. Hoegen, C.K. Renkamp, J. Liermann, C. Rippke, L. König and C. Buchele declare that they have no competing interests.

Open Access This article is licensed under a Creative Commons Attribution 4.0 International License, which permits use, sharing, adaptation, distribution and reproduction in any medium or format, as long as you give appropriate credit to the original author(s) and the source, provide a link to the Creative Commons licence, and indicate if changes were made. The images or other third party material in this article are included in the article's Creative Commons licence, unless indicated otherwise in a credit line to the material. If material is not included in the article's Creative Commons licence and your intended use is not permitted by statutory regulation or exceeds the permitted use, you will need to obtain permission directly from the copyright holder. To view a copy of this licence, visit http://creativecommons.org/licenses/by/4. $0 /$.

\section{References}

1. Ponti E, Lancia A, Ost P, Trippa F, Triggiani L, Detti B et al (2017) Exploring all avenues for radiotherapy in oligorecurrent prostate cancer disease limited to lymph nodes: a systematic review of the role of stereotactic body radiotherapy. Eur Urol Focus 3(6):538-544

2. Matsushita H, Jingu K, Umezawa R, Yamamoto T, Ishikawa Y, Takahashi N et al (2018) Stereotactic radiotherapy for oligometastases in lymph nodes-a review. Technol Cancer Res Treat 17: 1533033818803597

3. Vogelius IR, Bentzen SM (2013) Meta-analysis of the alpha/beta ratio for prostate cancer in the presence of an overall time factor: bad news, good news, or no news? Int J Radiat Oncol Biol Phys 85(1):89-94

4. Jereczek-Fossa BA, Ronchi S, Orecchia R (2015) Is stereotactic body radiotherapy (SBRT) in lymph node oligometastatic patients feasible and effective? Rep Pract Oncol Radiother 20(6):472-483

5. Noel CE, Parikh PJ, Spencer CR, Green OL, Hu Y, Mutic S et al (2015) Comparison of onboard low-field magnetic resonance imaging versus onboard computed tomography for anatomy visualization in radiotherapy. Acta Oncol 54(9):1474-1482

6. Van den Begin R, Engels B, Gevaert T, Duchateau M, Tournel K, Verellen D et al (2014) Impact of inadequate respiratory motion management in SBRT for oligometastatic colorectal cancer. Radiother Oncol 113(2):235-239

7. Bertholet J, Worm ES, Fledelius W, Hoyer M, Poulsen PR (2016) Time-resolved intrafraction target translations and rotations during stereotactic liver radiation therapy: implications for marker-based localization accuracy. Int J Radiat Oncol Biol Phys 95(2):802-809

8. Poulsen PR, Worm ES, Petersen JB, Grau C, Fledelius W, Hoyer M (2014) Kilovoltage intrafraction motion monitoring and target dose reconstruction for stereotactic volumetric modulated arc therapy of tumors in the liver. Radiother Oncol 111(3):424-430

9. Worm ES, Hoyer M, Fledelius W, Hansen AT, Poulsen PR (2013) Variations in magnitude and directionality of respiratory target motion throughout full treatment courses of stereotactic body radiotherapy for tumors in the liver. Acta Oncol 52(7):1437-1444 
10. Sterzing F, Brunner TB, Ernst I, Baus WW, Greve B, Herfarth K et al (2014) Stereotactic body radiotherapy for liver tumors. Strahlenther Onkol 190(10):872-881

11. van Sörnsen de Koste JR, Palacios MA, Bruynzeel AME, Slotman BJ, Senan S, Lagerwaard FJ (2018) MR-guided gated stereotactic radiation therapy delivery for lung, adrenal, and pancreatic tumors: a geometric analysis. Int J Radiat Oncol Biol Phys 102(4):858-866

12. Klüter S, Katayama S, Spindeldreier CK, Koerber SA, Major G, Alber M et al (2020) First prospective clinical evaluation of feasibility and patient acceptance of magnetic resonance-guided radiotherapy in Germany. Strahlenther Onkol 196:691-698

13. Tyran M, Cao M, Raldow AC, Dang A, Lamb J, Low DA et al (2018) Stereotactic magnetic resonance-guided online adaptive radiotherapy for oligometastatic breast cancer: a case report. Cureus 10(3):e2368

14. Guckenberger M, Baus WW, Blanck O, Combs SE, Debus J, Engenhart-Cabillic R et al (2020) Definition and quality requirements for stereotactic radiotherapy: consensus statement from the DEGRO/DGMP Working Group Stereotactic Radiotherapy and Radiosurgery. Strahlenther Onkol 196(5):417-420

15. Federmann JJLOV (2019) S3-Leitlinie Prostatakarzinom

16. Franzese C, Badalamenti M, Comito T, Franceschini D, Clerici E, Navarria P et al (2020) Assessing the role of stereotactic body radiation therapy in a large cohort of patients with lymph node oligometastases: Does it affect systemic treatment's intensification? Radiother Oncol 150:184-190

17. Franzese C, Comito T, Tripoli A, Franceschini D, Clerici E, Navarria $\mathrm{P}$ et al (2020) Phase II trial of high dose stereotactic body radiation therapy for lymph node oligometastases. Clin Exp Metastasis 37(5):565-573

18. Werensteijn-Honingh AM, Kroon PS, Winkel D, Aalbers EM, van Asselen B, Bol GH et al (2019) Feasibility of stereotactic radiotherapy using a $1.5 \mathrm{~T}$ MR-linac: multi-fraction treatment of pelvic lymph node oligometastases. Radiother Oncol 134:50-54

19. Winkel D, Bol GH, Werensteijn-Honingh AM, Intven MPW, Eppinga WSC, Hes J et al (2020) Target coverage and dose criteria based evaluation of the first clinical 1.5T MR-linac SBRT treatments of lymph node oligometastases compared with conventional CBCT-linac treatment. Radiother Oncol 146:118-125

20. Hanna G, Murray L, Patel R, Jain S, Aitken K, Franks K et al (2018) UK consensus on normal tissue dose constraints for stereotactic radiotherapy. Clin Oncol (R Coll Radiol) 30(1):5-14

21. Park C, Papiez L, Zhang S, Story M, Timmerman RD (2008) Universal survival curve and single fraction equivalent dose: useful tools in understanding potency of ablative radiotherapy. Int J Radiat Oncol Biol Phys 70(3):847-852

22. Winkel D, Kroon PS, Werensteijn-Honingh AM, Bol GH, Raaymakers BW, Jürgenliemk-Schulz IM (2018) Simulated dosimetric impact of online replanning for stereotactic body radiation therapy of lymph node oligometastases on the 1.5T MR-linac. Acta Oncol 57(12):1705-1712

23. Werensteijn-Honingh AM, Jürgenliemk-Schulz IM, Gadellaa-Van Hooijdonk CG, Sikkes GG, Vissers N, Winkel D et al (2020) Impact of a vacuum cushion on intrafraction motion during online adaptive MR-guided SBRT for pelvic and para-aortic lymph node oligometastases. Radiother Oncol 154:110-117
24. Jereczek-Fossa BA, Fanetti G, Fodor C, Ciardo D, Santoro L, Francia CM et al (2017) Salvage stereotactic body radiotherapy for isolated lymph node recurrent prostate cancer: single institution series of 94 consecutive patients and 124 lymph nodes. Clin Genitourin Cancer 15(4):e623-e632

25. Ingrosso G, Trippa F, Maranzano E, Carosi A, Ponti E, Arcidiacono F et al (2017) Stereotactic body radiotherapy in oligometastatic prostate cancer patients with isolated lymph nodes involvement: a two-institution experience. World J Urol 35(1):45-49

26. Yeung R, Hamm J, Liu M, Schellenberg D (2017) Institutional analysis of stereotactic body radiotherapy (SBRT) for oligometastatic lymph node metastases. Radiat Oncol 12(1):105

27. Witt JS, Rosenberg SA, Bassetti MF (2020) MRI-guided adaptive radiotherapy for liver tumours: visualising the future. Lancet Oncol 21(2):e74-e82

28. Corradini S, Alongi F, Andratschke N, Belka C, Boldrini L, Cellini $F$ et al (2019) MR-guidance in clinical reality: current treatment challenges and future perspectives. Radiat Oncol 14:92

29. Hall WA, Paulson ES, van der Heide UA, Fuller CD, Raaymakers B, Lagendijk JJ et al (2019) The transformation of radiation oncology using real-time magnetic resonance guidance: a review. Eur J Cancer 122:42-52

30. Finazzi T, Palacios MA, Spoelstra FOB, Haasbeek CJA, Bruynzeel AME, Slotman BJ et al (2019) Role of on-table plan adaptation in MR-guided ablative radiation therapy for central lung tumors. Int J Radiat Oncol Biol Phys 104(4):933-941

31. Finazzi T, Palacios MA, Haasbeek CJA, Admiraal MA, Spoelstra FOB, Bruynzeel AME et al (2020) Stereotactic MR-guided adaptive radiation therapy for peripheral lung tumors. Radiother Oncol 144:46-52

32. Palacios MA, Bohoudi O, Bruynzeel AME, van Sörsen de Koste JR, Cobussen P, Slotman BJ et al (2018) Role of daily plan adaptation in MR-guided stereotactic ablative radiation therapy for adrenal metastases. Int J Radiat Oncol Biol Phys 102(2):426-433

33. Padgett KR, Simpson G, Asher D, Portelance L, Bossart E, Dogan N (2020) Assessment of online adaptive MR-guided stereotactic body radiotherapy of liver cancers. Phys Med 77:54-63

34. El-Bared N, Portelance L, Spieler BO, Kwon D, Padgett KR, Brown KM et al (2019) Dosimetric benefits and practical pitfalls of daily online adaptive MRI-guided stereotactic radiation therapy for pancreatic cancer. Pract Radiat Oncol 9(1):e46-e54

35. Henke L, Kashani R, Yang D, Zhao T, Green O, Olsen L et al (2016) Simulated online adaptive magnetic resonance-guided stereotactic body radiation therapy for the treatment of oligometastatic disease of the abdomen and central thorax: characterization of potential advantages. Int J Radiat Oncol Biol Phys 96(5):1078-1086

36. Henke L, Kashani R, Robinson C, Curcuru A, DeWees T, Bradley J et al (2018) Phase I trial of stereotactic MR-guided online adaptive radiation therapy (SMART) for the treatment of oligometastatic or unresectable primary malignancies of the abdomen. Radiother Oncol 126(3):519-526

37. Chin S, Eccles CL, McWilliam A, Chuter R, Walker E, Whitehurst $P$ et al (2020) Magnetic resonance-guided radiation therapy: a review. J Med Imaging Radiat Oncol 64(1):163-177

38. Winkel D, Werensteijn-Honingh AM, Kroon PS, Eppinga WSC, Bol GH, Intven MPW et al (2019) Individual lymph nodes: "see it and zap it". Clin Transl Radiat Oncol 18:46-53 